\title{
Gas Emission Prediction Model of Coal Mine Based on CSBP Algorithm
}

\author{
Yan XIONG, Jia-Tang CHENG*,and Zhi-Mei DUAN \\ The Engineering College of Honghe University, Yunnan Mengzi 661199, China
}

\begin{abstract}
In view of the nonlinear characteristics of gas emission in a coal working face, a prediction method is proposed based on cuckoo search algorithm optimized BP neural network (CSBP). In the CSBP algorithm, the cuckoo search is adopted to optimize weight and threshold parameters of BP network, and obtains the global optimal solutions. Furthermore, the twelve main affecting factors of the gas emission in the coal working face are taken as input vectors of CSBP algorithm, the gas emission is acted as output vector, and then the prediction model of BP neural network with optimal parameters is established. The results show that the CSBP algorithm has batter generalization ability and higher prediction accuracy, and can be utilized effectively in the prediction of coal mine gas emission.
\end{abstract}

\section{Introduction}

Gas is one of the major natural disasters of the coal mine safety production, the security problem caused by it account to more than $80 \%$ of the coal mine safety in production accidents [1]. Therefore, accurate prediction gas emission is important to ensure the safety of the mine production and has practical significance. In recent years, in view of the gas emission prediction research, a series of models and methods are put forward by related personnel. $\mathrm{Fu}$ Hua et al. [2] used ant colony algorithm (ACO) to optimize the weights and threshold of the Elman neural network, and established the dynamic prediction method of absolute gas emission. Shi liang et al. [3] established gas emission prediction model based on the empirical mode decomposition (EMD), support vector machine (SVM) combined with particle swarm optimization algorithm (PSO). Fan Baolong et al. [4] used the local mean decomposition (LMD) method to get the production function component (PF component) and SVM model, and constructed the prediction model of gas emission in a coal working face.

However, due to the gas emission in working face is influenced by natural factors combine with mining technology, such as coal seam gas content, coal seam buried depth, coal seam thickness, coal seam dip angle and length of working face, complex nonlinear characteristics lie in them, so that the accurate prediction is difficult. Although the artificial neural network has good nonlinear mapping ability, it is easy to fall into local minimum point. Moreover, the parameters of the SVM algorithm are difficult to determine and affect the prediction precision [5].

As heuristic optimization algorithm, swarm intelligence optimization algorithm has been proposed in recent years. According to the natural biological behavior, some bionic algorithms have emerged such as genetic algorithm (GA), particle swarm optimization algorithm, ant colony algorithm, and Cuckoo Search (CS), etc. However, the genetic algorithm need to reproduction, crossover and mutation operations, and is prone to premature convergence. Ant colony algorithm programming is relatively complex and has low generality and then limits the scope of its application. The implementation process of particle swarm algorithm is simple. However, it is difficult to search for the global optimal solution and has other defects. As a kind of global searching method of intelligent optimization, the Cuckoo Search has the advantages of easy to implement, less parameter setting and so on. Studies show that the CS algorithm is superior to the GA algorithm and PSO algorithm [6,7]. Therefore, Cuckoo Search algorithm is combined with BP neural network to build a CSBP prediction model of gas emission, and the experimental data are used to validate the accuracy of the prediction results.

\section{Cuckoo Search Algorithm}

As a heuristic algorithm, cuckoo search [8] is put forward by Xin-she Yang and Suash Deb in 2009, which is used to simulate the parasitic brood behavior on the part of the Cuckoo. In the CS algorithm, the bird's nests are used to represent the solutions, and each of the cuckoo egg represents a data processing. The bad solutions are replaced with the new potential solutions. During the iteration process, the eggs with the best fitness will be held until the next generation. In addition, when the host finds exotic birds' eggs in the nest, they will abandon the exotic birds' eggs or build another new nest. According to the 
principle, the bird's nest is updated as follow:

$$
x_{i}^{k+1}=x_{i}^{k}+\alpha \oplus L(\lambda)
$$

Where, $k$ is the current iteration number, $\alpha$ donates the step length; $\oplus$ is entry-wise multiplication, $L(\lambda)$ donates random search path, and a random step length is produced with Levy distribution.

$$
L(\lambda) \sim u=t^{-\lambda}, \quad 1<\lambda \leq 3
$$

In addition, the parameter $p_{a}$ is set to simulate the probability of host finds exotic birds' eggs. A random number is used to compare with the discovery probability $p_{a}$, so as to determine whether or not generate new individual. At this point, location update formula is given as:

$$
x_{i}^{k+1}=x_{i}^{k}+\gamma \times H\left(p_{a}-\varepsilon\right) \oplus\left[x_{m}^{k}-x_{n}^{k}\right]
$$

Where, $\varepsilon$ and $\gamma$ are the random numbers generated uniformly in the interval $(0,1), H\left(p_{a}-\varepsilon\right)$ is Heaviside function.

In the CS algorithm, Levy flight consists in frequent short jumps and occasional long jump, and step length has randomness. In order to balance the global exploration and local development ability of the algorithm, the step length is adjusted dynamically, and given as:

$$
\alpha=\left(\alpha_{\max }-\alpha_{\text {min }}\right)\left(k / k_{\max }-1\right)^{2}+\alpha_{\text {min }}
$$

Where, $k_{\max }$ is the maximum number of iterations, $\alpha_{\max }$ and $\alpha_{\min }$ are the step size values of maximum and minimum.

At the same time, in order to enhance the diversity of population, a reverse learning strategy is used to search for the optimal position as much as possible. The one dimensional position of a bird's nest is chosen randomly at first, and then the dimension vector reversely is obtained to get a new site of bird's nest. At last, optimization operation is carried out according to the size of the fitness value. Reverse learning formula is:

$$
x_{i j}^{*}(k)=a_{j}(k)+b_{j}(k)-x_{i j}(k)
$$

Where, $x_{i j}^{*}(k)$ and $x_{i j}(k)$ donate the reverse learning position and the current position of nest, respectively; $a_{j}(k)$ and $b_{j}(k)$ are the $\mathrm{j}$ dimension border of the search space, respectively.

\section{CSBP Algorithm Implementation}

In the CSBP algorithm, each egg of cuckoo represents the initial weights and threshold of BP neural network, and CS algorithm is used to optimize the parameters. Then, the optimal solutions are transferred to the BP neural network and obtain the final output results. Implementation steps [9] are given as follows:

1) Initialize parameters of CS algorithm.

2) Generate initial solution randomly, and take as the weight and threshold parameters of BP network.

3) Choose the best fitness and the best position of the bird's nests.

4) According to the formula (1), the position of new bird's nests is calculated, and according to the size of the fitness value to keep good position and fitness of bird's nest.

5) Using random number $\varepsilon$ compare to discovery probability $p_{a}$, the part of the original individuals are abandoned by formula (3). At the same time, the new nests are generated. Then the bad bird's nests are replaced, and the fitness is updated subsequently.

6) To judge whether meet the condition of algorithm to stop, if it meet the condition then turn into the next step, or transfer into step 4) to continue.

7) Output the optimal position of the bird's nests, and they are taken as the initial parameters of weight and threshold of BP network. Finally, the learning and training are complimented through the BP network.

\section{Gas Emission Prediction}

\subsection{Parameters Selection}

The influence factors of gas emission in coal working face are numerous, and seriously affected by the gas content in coal seam and its occurrence conditions. Therefore, how to correctly select these factors is the precondition to establishment the prediction model of gas emission. Research shows that the main influencing factors of gas emission $\mathrm{Y}\left(\mathrm{m}^{3} / \mathrm{t}\right)$ in working face are: coal seam gas content $X_{1}\left(m^{3} / t\right)$, buried depth of coal seam $X_{2}(m)$, coal seam thickness $\mathrm{X}_{3}(\mathrm{~m})$, dip Angle of coal seam $\mathrm{X}_{4}\left({ }^{\circ}\right)$, working face length $\mathrm{X}_{5}(\mathrm{~m})$, propulsion speed $\mathrm{X}_{6}(\mathrm{~m} / \mathrm{min})$, extraction rate $X_{7}$, adjacent layer gas content $X_{8}\left(\mathrm{~m}^{3} / \mathrm{t}\right)$, adjacent layer thickness $\mathrm{X}_{9}(\mathrm{~m})$, the distance between adjacent layers $\mathrm{X}_{10}(\mathrm{~m})$, interlayer litho $\operatorname{logy} \mathrm{X}_{11}$ and mining depth $\mathrm{X}_{12}(\mathrm{~m})$. Despite the roof management mode has a great influence on the gas emission, the roof management style of mining working face is the equal value, so it is not considered. Specific data [10] is shown in Table 1. 
Table 1 The statistical data

\begin{tabular}{cccccccccccccc}
\hline No. & $\mathrm{X}_{1}$ & $\mathrm{X}_{2}$ & $\mathrm{X}_{3}$ & $\mathrm{X}_{4}$ & $\mathrm{X}_{5}$ & $\mathrm{X}_{6}$ & $\mathrm{X}_{7}$ & $\mathrm{X}_{8}$ & $\mathrm{X}_{9}$ & $\mathrm{X}_{10}$ & $\mathrm{X}_{11}$ & $\mathrm{X}_{12}$ & $\mathrm{Y}$ \\
\hline 1 & 1.92 & 408 & 2.0 & 155 & 155 & 4.42 & 0.960 & 2.02 & 1.50 & 20 & 5.03 & 1825 & 3.34 \\
2 & 2.15 & 411 & 2.0 & 140 & 140 & 4.16 & 0.950 & 2.10 & 1.21 & 22 & 4.87 & 1527 & 2.97 \\
3 & 2.14 & 420 & 1.8 & 175 & 175 & 4.13 & 0.950 & 2.64 & 1.62 & 19 & 4.75 & 1751 & 3.56 \\
4 & 2.58 & 432 & 2.3 & 145 & 145 & 4.67 & 0.950 & 2.40 & 1.48 & 17 & 4.91 & 2078 & 3.62 \\
5 & 2.40 & 456 & 2.2 & 160 & 160 & 4.51 & 0.940 & 2.55 & 1.75 & 20 & 4.63 & 2104 & 4.17 \\
6 & 3.22 & 516 & 2.8 & 180 & 180 & 3.45 & 0.930 & 2.21 & 1.72 & 12 & 4.78 & 2242 & 4.60 \\
7 & 2.80 & 527 & 2.5 & 180 & 180 & 3.28 & 0.940 & 2.81 & 1.81 & 11 & 4.51 & 1979 & 4.92 \\
8 & 3.35 & 531 & 2.9 & 165 & 165 & 3.68 & 0.930 & 1.88 & 1.42 & 13 & 4.82 & 2288 & 4.78 \\
9 & 3.61 & 550 & 2.9 & 155 & 155 & 4.02 & 0.920 & 2.12 & 1.60 & 14 & 4.83 & 2325 & 5.23 \\
10 & 3.68 & 563 & 3.0 & 175 & 175 & 3.53 & 0.940 & 3.11 & 1.46 & 12 & 4.53 & 2410 & 5.56 \\
11 & 4.21 & 590 & 5.9 & 170 & 170 & 2.85 & 0.795 & 3.40 & 1.50 & 18 & 4.77 & 3139 & 7.24 \\
12 & 4.03 & 604 & 6.2 & 180 & 180 & 2.64 & 0.812 & 3.15 & 1.80 & 16 & 4.70 & 3354 & 7.80 \\
13 & 4.34 & 607 & 6.1 & 165 & 165 & 2.77 & 0.785 & 3.02 & 1.74 & 17 & 4.62 & 3087 & 7.68 \\
14 & 4.80 & 634 & 5.5 & 175 & 175 & 2.92 & 0.773 & 2.98 & 1.92 & 15 & 4.55 & 3620 & 8.51 \\
15 & 4.67 & 640 & 6.3 & 175 & 175 & 2.75 & 0.802 & 2.56 & 1.75 & 15 & 4.60 & 3412 & 7.95 \\
16 & 2.43 & 450 & 2.2 & 160 & 160 & 4.32 & 0.950 & 2.00 & 1.70 & 16 & 4.84 & 1996 & 4.06 \\
17 & 3.16 & 544 & 2.7 & 165 & 165 & 3.81 & 0.930 & 2.30 & 1.80 & 13 & 4.90 & 2207 & 4.92 \\
18 & 4.62 & 629 & 6.4 & 170 & 170 & 2.80 & 0.803 & 3.35 & 1.61 & 19 & 4.63 & 3456 & 8.04 \\
\hline
\end{tabular}

\subsection{Model Establishment}

According to the actual data of coal mine gas emission in table 1, the first 14 groups as training samples are selected after the normalized processing, the last four groups are taken as the test data, and the prediction model of gas emission is establish based on CSBP algorithm. The main parameters are given as follows: the topology of the BP neural network is $12-15-1$, learning rate 0.1 , vector error convergence factor $10^{-5}$, the initial population size 25 , the maximum number of iterations $k_{\max }=100$, the initial step length $a_{\max }=1, a_{\min }=0.1$, limiting range of optimal parameters $[-5,5]$, the discovery probability $p_{a}=0.25$. According to the training data, the curve of the fitness function of the CSBP algorithm is shown in Figure 1.

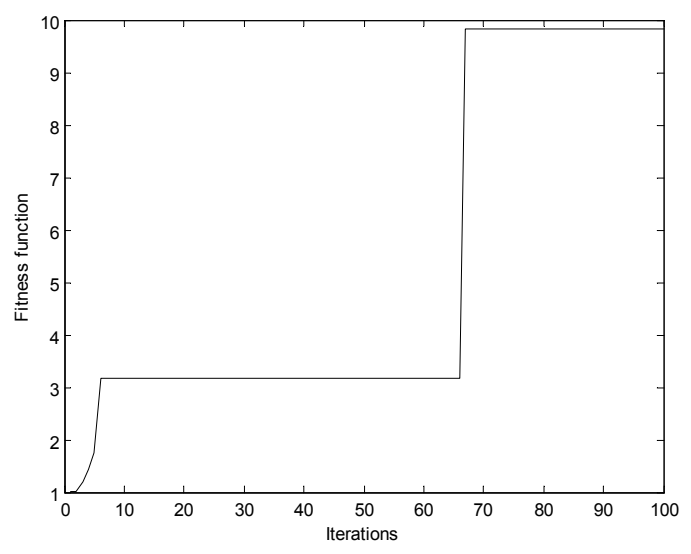

Figure 1 The curve of fitness function

It can be seen from Figure 1, the fitness value of CS algorithm is increased gradually from 0 , the iteration number is about 7 times or so, the fitness function curve entries into a longer flat area, it is illustrated that the algorithm falls into local optimum. Due to Levy flight strategy in the CS algorithm consists of frequent short leap and occasional long jump, when iterative 67 times, the value of fitness function increases again, the algorithm has been jump out of the local optimal area, and search continually the optimal solution in the solution space, and the fitness value steady at 9.83 finally. Therefore, the CS algorithm has the good capability of local exploration, and can be applied effectively to the parameters optimization system of BP neural network.

\subsection{Result Analysis}

The last 4 groups' sample data is used to forecast the gas emission based on the CSBP algorithm, and the results are compared with BP neural network prediction outputs, the comparison is shown in Table 2. It is shown that maximum the relative error of CSBP algorithm is $4.78 \%$, the average relative error (ARE) is $2.435 \%$. And the maximum relative error of BP neural network is $10.34 \%$, the average relative error is $7.49 \%$. Obviously, comparing with BP neural network, the CSBP algorithm has higher prediction precision which can better meet the engineering needs of gas emission prediction.

Table 2 The prediction effects contrast and analysis

\begin{tabular}{llllll}
\hline & \multirow{2}{*}{$\begin{array}{l}\text { The } \\
\text { actual } \\
\text { value }\end{array}$} & \multicolumn{3}{c}{ CSBP prediction } & \multicolumn{2}{c}{ BP prediction } \\
\cline { 3 - 6 } & value & $\begin{array}{l}\text { The } \\
\text { relative } \\
\text { error } / \%\end{array}$ & $\begin{array}{l}\text { Predictive } \\
\text { value }\end{array}$ & $\begin{array}{l}\text { The } \\
\text { relative } \\
\text { error } / \%\end{array}$ \\
\hline 15 & 7.95 & 8.33 & 4.78 & 7.29 & 8.30 \\
16 & 4.06 & 3.94 & 2.96 & 4.48 & 10.34 \\
17 & 4.92 & 4.92 & 0 & 4.73 & 3.86 \\
18 & 8.04 & 7.88 & 2.00 & 7.40 & 7.46 \\
\hline \multicolumn{3}{l}{ ARE $/ \%$} & 2.435 & & 7.49 \\
\hline
\end{tabular}

In addition, as a variable to measure the difference between actual value with predictive value, the generalization ability of neural network can be used the average relative variation (ARV). The smaller value is, the stronger generalization ability of the network obtains. The formula is given as: 


$$
A R V=\frac{\sum_{i=1}^{n}[Y(i)-\hat{Y}(i)]^{2}}{\sum_{i=1}^{n}[Y(i)-\bar{Y}(i)]^{2}}
$$

Where, $Y(i), \hat{Y}(i)$ and $\bar{Y}(i)$ are the actual, forecast and average value of gas emission, respectively.

By calculation, the AVR of the CSBP and BP neural network are $1.46 \%$ and $8.36 \%$, respectively. Obviously, the CSBP algorithm enhances the generalization ability of neural network.

\section{Summary}

The influence factors of gas emission in a coal working face are numerous, so the prediction model of gas emission based on CSBP is proposed. The CS algorithm is used to optimist the weights and threshold parameters of BP neural network, and avoids the defect of the BP network is easy to fall into local minimum.

According to the test samples of gas emission, the results show that the average relative error based on CSBP algorithm is $2.435 \%$, the average relative variation is $1.46 \%$, which is less than the corresponding value of the BP neural network, and a new idea is provided for gas emission prediction in a coal mine working face.

\section{References}

1. LIU June, AN Fengping, LIN Dachao, et al, Prediction of gas emission from coalface by intrinsic mode SVM modeling, Systems Engineering-Theory \& Practice, 33(2), 505-511 (2013)

2. FU Hua, XIE Sen, XU Yaosong, et al, Gas emission dynamic prediction model of coal mine based on ACC-ENN algorithm, Journal of China Coal Society, 39(7), 1296-1301 (2014)

3. SHI Shiliang, LI Runqiu, LUO Wenke, Method for predicting coal mine gas emission based on EMD-PSO-SVM and its application, China Safety Science Journal, 24(7), 43-49 (2014)

4. FAN Baolong, BAI Chunhua, LI Jianping, Forecasting model of coalface gas emission based on LMD-SVM method, Journal of Mining \& Safety Engineering, 30(6), 946-952 (2013).

5. CHENG Jiatang, DUAN Zhimei, XIONG Yan, QAPSO-BP algorithm and its application in vibration fault diagnosis for a hydroelectric generating unit, JOURNAL OF VIBRATION AND SHOCK, 34(23), 177-181, 201 (2015)

6. VALIAN E, MOHANNA S, TAVAKOLI S, Improved cuckoo search algorithm for feedforward neural network training, Int J Artif Intell Appl, 2( 3) , 36-43 (2011)

7. Sadollah A, Bahreininejad A, Eskandar H, Mine blast algorithm: A new population based algorithm for solving constrained engineering optimization problems, Applied Soft Computing, 13(5), 2592-2612 (2013)

8. Thang Trung Nguyen, Dieu Ngoc Vo, Bach Hoang
Dinh, Cuckoo search algorithm for combined heat and power economic dispatch, Electrical Power and Energy Systems, (81), 204-214 (2016)

9. SHEN Mingke, HUANG Zhenyu, WANG Zhihua, et al, Prediction of coal ash deformation temperature based on Cuckoo Search and BP Neural Network, Journal of Fuel Chemistry and Technology, 42(12), 1423-1430 (2014)

10. Lü Fu, LIANG Bing, SUN Weiji, et al, Gas emission quantity prediction of working face based on principal component regression analysis method, Journal of China Coal Society, 37(1), 113-116 (2012) 